\title{
Réaliser la Vision de la SCPH en regard de la pharmacie hospitalière au Canada
}

par Patricia Macgregor

L a profession de pharmacien est en pleine mutation de son champ d'exercice. En effet, les gouvernements provinciaux, en collaboration avec les ordres de pharmaciens, sont en train de légiférer pour encadrer le champ d'exercice élargi des pharmaciens et de réglementer les techniciens en pharmacie. Ces deux professionnels ont des occasions d'élargir leurs rôles au sein de l'équipe des soins de santé.

Nous avons demandé à élargir notre champ de pratique et la réponse a été favorable, mais saisissons-nous les nouvelles occasions que cela offre? Combien de pharmaciens recevront l'attestation de formation pour administrer des injections? Combien ajusteront des ordonnances? Quel pourcentage d'assistants techniques en pharmacie embrasseront la réglementation et exerceront pleinement leur pratique? Les questions sont en effet nombreuses. Les ministères de la Santé des provinces nous ont témoigné leur confiance, mais sommes-nous prêts à satisfaire pleinement les critères que cela implique? Nous pouvons certainement tirer des leçons de nos collègues d'autres professions qui sont passés par là avant nous. Par exemple, les infirmières et infirmiers et les inhalothérapeutes ont rapidement adopté leur champ d'exercice élargi (dont l'activité de prescription) dans les hôpitaux et au sein des groupes de médecine de famille et des services de soins à domicile. L'adoption par les pharmaciens des activités ajoutées à leur champ d'exercice est l'occasion d'accroître la visibilité de notre profession aux yeux du public, des équipes de soins de santé, des ministères de la Santé et d'autres parties prenantes clés.

En plus de créer de nouveaux rôles, ces changements engendrent aussi des exigences et des pressions supplémentaires. $\mathrm{La}$ SCPH vient aider ses membres à composer avec ces nouvelles exigences et à faire reconnaître la valeur de notre travail dans le cadre des soins aux patients. Plus particulièrement, le projet SCPH 2015 propose d'excellentes activités mesurables qui représentent des occasions non seulement de bonifier notre pratique, mais aussi de faire connaître la portée de notre travail au sein de nos organisations respectives, et au-delà. Les indicateurs de performance clinique clés, qui sont actuellement développés au moyen d'une méthode Delphi robuste et qui seront présentés à la Conférence sur la pratique professionnelle (CPP) en février 2013, nous permettront précisément de faire la promotion de la valeur de nos activités cliniques. Par ailleurs, le comité de valorisation de la SCPH souligne diligemment la valeur des pharmaciens dans le cercle des soins de santé aux parties prenantes clé. La SCPH répond également aux besoins changeants de ses membres en matière de communication, de participation et d'apprentissage en créant ou en élargissant les voies de communication électroniques. Vous pouvez donc maintenant joindre la SCPH sur Facebook, participer au projet SCPH 2015 en vous exprimant dans notre blogue et en nous suivant sur Twitter, et tirer profit de divers webminaires éducatifs. Dans le cadre de la CPP, soyez à l'affût des projets de résidence primés, des présentations par affiches virtuelles et des contributions au concours de vidéos pour étudiants; et pourquoi ne pas songer à poser votre candidature pour les prix l'an prochain?

Vous pouvez aussi réseauter et échanger avec des collègues sur l'un des 23 réseaux de spécialistes en pharmacie (RSP) de la $\mathrm{SCPH}$, qui couvrent divers domaines, comme la gestion responsable des antimicrobiens, l'utilisation des médicaments, les urgences, les soins à domicile, la pédiatrie et la greffe. Il y a même un RSP précisément pour les pharmaciens qui exercent dans les petits hôpitaux. Les membres qui y participent considèrent les RSP comme très informatifs en matière de nouvelles pratiques et lignes directrices, d'idées sur la façon de mieux servir les patients et de partager les connaissances, de communication avec les pairs de tout le Canada, etc. Quelle meilleure façon d'entrer en relation de façon efficace et productive avec des collègues ayant des intérêts et des compétences similaires qu'ils veulent partager? Visitez le www.cshp.ca pour plus d'information.

Les pharmaciens sont des professionnels créatifs, novateurs, dévoués, empressés de collaborer afin de faire évoluer les soins aux patients. Je vous invite à prendre l'initiative : participez, soyez des leaders, engagez-vous et faites la promotion de notre profession.

[Traduction par l'éditeur]

Patricia Macgregor, B. Sc., R. Ph., M. R. Pharm. S., M. H. Sc., C.H.E., est présidente désignée et agente de liaison pour la Société canadienne des pharmaciens d'hôpitaux. 